\title{
Célia Mello: contribuição acadêmica na formação de recursos humanos.
}

\author{
A.G. Luiz, C.N. Campos, J.F.M. Oliveira \\ L.A.P. Lozada, R.L.M. Vulcani, S.M. de Almeida, \\ S. Dantas, V. Pedrotti
}

\begin{abstract}
Neste artigo prestamos homenagem à professora Célia Picinin de Mello por ocasião dos seus 70 anos, relatando uma pequena parte da sua história acadêmica com relação à formação de recursos humanos durante o seu trabalho como professora e pesquisadora na Universidade Estadual de Campinas (UNICAMP).
\end{abstract}

\section{Introdução}

Através de seu trabalho acadêmico, a professora e pesquisadora Célia Picinin de Mello contribuiu significativamente para a área de Ciência da Computação, tanto na produção de importantes resultados científicos, como na formação de recursos humanos.

No seu período de credenciamento na pós-graduação do Instituto de Computação da Universidade Estatual de Campinas, Célia de Mello orientou 8 dissertações de mestrado, 3 teses de doutorado e 1 supervisão de pós-doutorado. Neste artigo, alguns dos seus ex-alunos escrevem pequenos

2000 AMS Subject Classification: 01A70, 05Cxx.

Keywords and Phrases: homenagem, história acadêmica, teoria dos grafos. 
depoimentos sobre a sua tragetória acadêmica e prestam homenagens a esta grande pessoa e profissional.

\section{Atílio Gomes Luiz}

- Afiliação: Universidade Federal do Ceará, CE.

- Vínculo com Célia: orientadora do mestrado.

Iniciei o mestrado na UNICAMP no segundo semestre de 2011 e cursei, dentre várias disciplinas, a disciplina de Teoria dos Grafos, ministrada pela profa. Célia P. de Mello. Logo após a disciplina, iniciei os estudos do mestrado sob a orientação da Célia e sob coorientação da profa. Christiane Neme Campos. Inicialmente, minha pesquisa de mestrado foi financiada pela CAPES e, posteriormente, pela FAPESP. Como problema de pesquisa do mestrado, a Célia me apresentou uma variante da coloração total que, em português, batizamos como coloração total semiforte, definida logo a seguir. Uma coloração total $\varphi$ de um grafo sem laços $G$ é uma atribuição de cores aos vértices e às arestas de $G$ de modo que quaisquer dois elementos adjacentes ou incidentes possuam cores distintas. Para cada vértice $v \in$ $V(G)$, seja $C(v)=\{\varphi(v)\} \cup\{\varphi(u v): u v \in E(G)\}$ o conjunto de cores formado pela cor do vértice $v$ e pelas cores das arestas que nele incidem. Dizemos que a coloração total $\varphi$ é semiforte se $C(u) \neq C(v)$ para quaisquer dois vértices adjacentes $u, v \in V(G)$. O número cromático total semiforte de $G$ é o menor número de cores para o qual $G$ possui uma coloração total semiforte.

A principal conjetura envolvendo esta coloração afirma que o número cromático total semiforte de todo grafo $G$ sem laços é no máximo $\Delta(G)+3$. Durante o meu mestrado, verificamos essa conjetura para a classe dos grafos equipartidos completos [2], assim como também determinamos o número cromático total semiforte para a classe dos grafos subcúbicos sem vértices adjacentes de grau máximo e várias classes de grafos cúbicos [3]. Agradeço à Célia pela atenção e dedicação que ela sempre demonstrou durante minha convivência com ela, que me inspiram até hoje. 


\section{Christiane Neme Campos}

- Afiliação: Universidade Estadual de Campinas, SP.

- Vínculo com Célia: orientadora do doutorado.

Conheci a Célia como professora da disciplina de Teoria de Grafos quando iniciei a Pós-Graduação. Em 2000, ela se tornou minha orientadora de doutorado. Foi um período de muito aprendizado, um aprendizado que extrapola o conhecimento técnico. O aprendizado técnico está expresso nos trabalhos que publicamos juntas [1, 2, 3, 4, 5, 6, 7, 8, seja como sua aluna de doutorado ou, posteriormente, como sua colega de instituto. O aprendizado não técnico, carrego comigo e transmito aos meus alunos, passados e futuros, na tentativa de que eu possa ser para eles, pelo menos, um pouco do que ela foi e é para mim.

\section{José Francisco Mota Oliveira}

- Afiliação: Escola Estadual Professor Ernani Calbucci, SP

- Vínculo com Célia: orientadora do mestrado.

A profa. Anamaria Gomide foi minha orientadora de Mestrado Profissional na Unicamp em 2014. Foi através dela que tive o prazer e privilégio de conhecer e ter a profa. Célia como Orientadora na dissertação do tema "Tesselações do Plano Euclidiano". A orientação da Célia, além de ter me transformado no quesito das competências técnicas de professor, também contribuiu na minha formação humana, pois foram momentos preciosos de boas conversas, risadas e acima de tudo um amor e paciência na arte de ensinar. Enfim, gratidão por tudo que me proporcionou como profissional e como ser humano querida e prezada Célia. 


\section{Luis Arturo Perez Lozada}

- Afiliação: Pesquisador Independente.

- Vínculo com Célia: orientadora do Mestrado.

Meu vínculo acadêmico com a Profesora Célia Picinin de Mello começou na disciplina de Teoria dos Grafos ministrada por ela no ano de 1995. Como parte da dinâmica de ensino-apreendizagem de tal disciplina a Professora Célia propôs o estudo de diversos tópicos sobre classes de Grafos. Nessas apresentações conheci o operador Clique e os Grafos Clique-Convergentes. Logo após finalizar a disciplina, pedi à Professora Célia para ser minha orientadora de Mestrado. Tal pedido e sua aceitação foram um agrado mútuo. Durante nossas pesquisas discorremos sobre diversas pesquisas relacionados aos Grafos Clique e elaboramos uma dissertação a maneira de compendio sobre esta classe de Grafos. Em essência cada capítulo da dissertação aborda um tópico relacionado aos Grafos Clique; vale ressaltar os capítulos que apresentam estudos das propriedades dos grafos clique em conhecidas classes de grafos; a clique-convergência; a clique-divergência e o diâmetro dos grafos clique-iterados.

\section{$5 \quad$ Renata de Lacerda Martins Vulcani}

- Afiliação: Escola Estadual Padre Armani, SP.

- Vínculo com Célia: orientadora do mestrado.

Conheci a Professora Célia através da Professora Anamaria Gomide, a qual me deu aula no Mestrado Profissional na UNICAMP. Quando decidimos que o tema da minha dissertação seria Grafos, ela disse: "Conheço alguém que vai nos ajudar muito!" e me apresentou para a querida Professora Célia! Simpática e atenciosa desde o primeiro encontro, a Célia nos recebia em sua sala no IMECC todas as quartas feiras a tarde do ano de 2014 para me orientar na elaboração da dissertação de mestrado. Posso dizer sem medo de errar que, muito mais do que sobre grafos eulerianos, 
aprendi com ela sobre profissionalismo, empatia e solidariedade. Por isso serei eternamente grata!

\section{Sheila Moraes de Almeida}

- Afiliação: Universidade Tecnológica Federal do Paraná, PR.

- Vínculo com Célia: orientadora de Trabalho de Conclusão de Curso na Graduação, de Mestrado e de Doutorado.

Célia foi minha professora na disciplina de Análise de Algoritmos I, na Universidade Estadual de Campinas, durante a graduação em Ciência da Computação. Neste primeiro contato, sua forma de apresentar o tema, com tanta clareza e didática, despertaram em mim grande interesse pela área. Então, no segundo semestre de 2001, decidi cursar mais uma disciplina ministrada por ela, Teoria dos Grafos. Apaixonei-me pelos grafos e pedi à Professora Célia que me orientasse em meu Trabalho de Conclusão de Curso (TCC). Estive sob orientação dela nesses quase 20 anos: depois do TCC, no mestrado e, depois durante o doutorado. Célia tornou-se uma grande amiga; sua alegria e simpatia iluminam os dias de todos à sua volta. No TCC e, mais tarde, no mestrado com a coorientação da Professora Anamaria Gomide, estudamos a caracterização e o reconhecimento dos Grafos Ponto-Intervalo, mais conhecidos como Grafos PI [9]. Um grafo $P I, G$, é um grafo interseção de uma família de triângulos entre duas retas paralelas, $R_{1}$ e $R_{2}$, tal que todo triângulo tem um vértice em $R_{1}$, um lado em $R_{2}$, e corresponde a um vértice de $G$; existe aresta entre dois vértices de $G$ se e somente se os triângulos correspondentes têm interseção não-vazia. Nós identificamos relações da classe dos grafos PI com outras classes de grafos bem conhecidas, como grafos de intervalos, permutação e co-comparabilidade. Também encontramos propriedades comuns com outras classes menos estudadas, mas que possuem estruturas muito similares a dos grafos PI, como os grafos interseção de trapézios, de paralelogramos, e os PI*. Provamos que a inclusão da classe PI em 
$\mathrm{PI}^{*}$ é estrita e que todo grafo PI que não é um grafo de intervalos possui um $C_{4}$ como subgrafo induzido. Apresentamos uma caracterização para os grafos PI, impondo restrições à sua representação através da interseção de uma família de trapézios entre duas retas paralelas. E exibimos a família dos subgrafos proibidos para a classe dos grafos PI que não são grafos permutação. No doutorado, sob coorientação da Professora Aurora Morgana, da Universidade de Roma - La Sapienza, estudamos o Problema da Coloração de Arestas em Grafos Split [10]. O Problema da Coloração de Arestas é determinar o menor número de cores com que se pode colorir as arestas de um dado grafo $G$ de maneira que quaisquer duas arestas que compartilham um vértice tenham cores distintas; esse número é chamado de índice cromático de $G$ e denotado por $\chi^{\prime}(G)$. Por definição, $\chi^{\prime}(G) \geq \Delta(G)$ para qualquer grafo simples $G$, onde $\Delta(G)$ é o maior número de arestas incidentes em um mesmo vértice de $G$. Em 1964, Vizing [11] provou que $\chi^{\prime}(G) \leq \Delta(G)+1$ para qualquer grafo simples $G$. Mesmo assim, decidir se um grafo simples $G$ tem $\chi^{\prime}(G)=\Delta(G)$ é um problema NP-completo, ainda que restrito aos grafos perfeitos ou aos grafos de comparabilidade [12]. Um grafo é split se e somente se seu conjunto de vértices pode ser particionado em uma clique e um conjunto independente. Chen e outros [13] provaram que se $G$ é split e tem $\Delta(G)$ ímpar, então $\chi^{\prime}(G)=\Delta(G)$. Seja $G$ um grafo split com uma partição do conjunto de vértices em uma clique $Q$ e um conjunto independente $S$. Provamos que se $S$ contém um vértice com grau no mínimo $|Q| / 2$ e no máximo $\Delta(G) / 2$, então $\chi^{\prime}(G)=\Delta(G)$. Apresentamos uma caracterização estrutural dos grafos split que conhecidamente têm $\chi^{\prime}(G)=\Delta(G)+1$. Também desenvolvemos algoritmos polinomiais que apresentam uma coloração de arestas com $\Delta(G)$ cores para um subconjunto dos grafos split que tem $\Delta(G)$ par.

\section{$7 \quad$ Simone Dantas}

- Afiliação: Universidade Federal Fluminense, RJ.

- Vínculo com Célia: supervisão de Pós-doutorado 
Após a conclusão do doutorado em 2002, iniciei o Pós-doutorado na UNICAMP, com financiamento do CNPq, e sob a supervisão de Célia P. de Mello. Inicialmente Célia me apresentou seu aluno de iniciação científica Luis Menegheti, e assim tive a primeira experiência de coorientação. O resultado do trabalho foi apresentado no Workshop on Graph Theory and Applications [17]. Concomitantemente, trabalhamos com sua aluna de doutorado Christiane N. Campos, atualmente professora da UNICAMP, em um problema de coloração associado às arestas de hipergrafos [3, 15].

Por ocasião da visita da pesquisadora Aurora Morgana (Universitá degli Studi di Roma) à UNICAMP, unimos dois temas de nosso interesse: problemas grafo sanduíche (estudados anteriormente em minha tese de doutorado), e a classe de grafos P4 esparsos (estudados por Sulamita Klein (COPPE/UFRJ), Morgana e Mello) [14, 16]. Mesmo após o término da bolsa, continuamos a nossa colaboração em pesquisa. Nosso último trabalho foi o estudo de um problema surgido a partir da tese de Campos, que tratava do número cromático total de famílias de Snarks [5] e que foi um momento de grande aprendizado.

Agradeço a generosidade e a atenção da Célia pelas inúmeras oportunidades que me proporcionou e que contribuíram para a formação da pesquisadora e da pessoa que sou hoje.

\section{Vagner Pedrotti}

- Afiliação: Universidade Federal de Mato Grosso do Sul, MS.

- Vínculo com Célia: orientadora do mestrado e do doutorado.

Cursei a disciplina de teoria dos grafos ministrada pela Célia na Unicamp e, após este primeiro contato com o assunto, ela aceitou ser minha orientadora de mestrado em 2005. Nos dois anos seguintes estudamos algoritmos para decomposição modular e as classes de grafos com poucos $P_{4}$ 's $\left(P_{4}\right.$-reduced, $P_{4}$-sparse, $P_{4}$-lite, $P_{4}$-extendible, $P_{4}$-tidy, $P_{4}$-laden, entre outras), produzindo uma dissertação na forma de survey sobre as várias 
decomposições e problemas resolvidos eficientemente em cada classe. A orientação da Célia nestes anos contribuiu significantemente para o avanço do trabalho, dado o seu conhecimento prévio de várias das classes.

Em seguida, entre 2007 e 2011, cursei meu doutorado na Unicamp, novamente sob a orientação da Célia. Inicialmente, continuamos o estudo das mesmas classes de grafos, agora expandindo o estudo para novos problemas e propomos um algoritmo para computar os Separadores Minimais de grafos extended $P_{4}$-laden [20] e outro para computar o empacotamento de cliques em grafos $P_{4}$-tidy [18. Então, decidimos estudar um tópico diverso e demonstramos limitantes superiores para a coloração total forte dos grafos indiferença [19]. Novamente, foi importante a experiência anterior da Célia com coloração total e com algoritmos para grafos indiferença para a escolha deste problema e o trabalho realizado.

Sou muito grato por ter estudado com Célia todos estes anos, desde o contato inicial com grafos numa disciplina excelente até as inúmeras contribuições e inspirações que ela apresentava em nosso trabalho. 


\section{References}

[1] A. G. Luiz, C. N. Campos e C. P. de Mello. AVD-total-chromatic number of some families of graphs with $\Delta(G)=3$. Discrete Applied Mathematics, 217(2017), pp. 628-638.

[2] A. G. Luiz, C. N. Campos e C. P. de Mello. AVD-total-colouring of complete equipartite graphs. Discrete Applied Mathematics, 184(2015), pp. 189-195.

[3] C. N. Campos, S. Dantas e C. P. de Mello. Colouring CliqueHypergraphs of Circulant Graphs. Graphs and Combinatorics, 29(2013), pp. 1713-1720.

[4] C. N. Campos, C. H. de Figueiredo, R. Machado e C. P. de Mello. The total chromatic number of split-indifference graphs. Discrete Mathematics, 312(2012), pp. 2690-2693.

[5] C. N. Campos, S. Dantas e C. P. de Mello. The total-chromatic number of some families of snarks. Discrete Mathematics, 311(2011), pp. 984-988.

[6] C. N. Campos e C. P. de Mello. The total chromatic number of some bipartite graphs. Ars Combinatoria, 88(2008), pp. 335-347.

[7] C. N. Campos e C. P. de Mello. A result on the total colourings of powers of cycles. Discrete Applied Mathematics, 155(2007), pp. 585597.

[8] C. N. Campos e C. P. de Mello. Coloração total do $C_{n}^{2}$. Tendências em Matemática Aplicada e Computacional, 4(2003), pp. 177-186.

[9] Sheila M. Almeida. Grafos PI. Dissertação de Mestrado. Universidade Estadual de Campinas, 2005.

[10] Sheila M. Almeida. Coloração de Arestas de Grafos Split. Tese de Doutorado. Universidade Estadual de Campinas, 2012. 
[11] V. G. Vizing. On an estimate of the chromatic class of a p-graph. Diskret. Analiz.1, 3:25-30, 1964.

[12] L. Cai e J. A. Ellis. Np-completeness of edge-colouring some restricted graphs. Discrete Applied Mathematics, 30:15-27, 1991.

[13] B.-L. Chen, H.-L. Fu, e M. T. Ko. Total chromatic number and chromatic index of split graphs. Journal of Combinatorial Mathematics and Combinatorial Computing, 17:137-146, 1995.

[14] S. Dantas, S. Klein, C. P. de Mello, A. Morgana. The Graph Sandwich Problem for P4-sparse graphs. Discrete Mathematics, v. 309, p. 36643673, 2009.

[15] C. N. Campos, S. Dantas, C. P. de Mello. Colouring cliquehypergraphs of circulant graphs. In: IV Latin-American Algorithms, Graphs and Optimization Symposium (LAGOS'07), 2008, Puerto Varas. Electronic Notes in Discrete Mathematics, 2008. v. 30. p. 189194.

[16] S. Dantas, S. Klein, C. P. de Mello, A. Morgana. The P4-sparse Graph Sandwich Problem. In: 7th International Colloquium on Graph Theory (ICGT'05), 2005, Hy $\tilde{A}$ “res. Electronic Notes on Discrete Mathematics, 2005. v. 22. p. 185-188.

[17] S. Dantas, C. P. Mello, L. F. A. B. Menegheti. Coloring the cliquehypergraphs of power of cycles. In: Workshop on Graph Theory and Applications, 2006, Porto Alegre. Workshop on Graph Theory and Applications, 2006.

[18] V. Pedrotti and C. P. de Mello. kr-packing of p4-tidy graphs. Technical Report 9-19, Instituto de Computação, Universidade de Campinas, 2009.

[19] V. Pedrotti and C. P. de Mello. Adjacent-vertex-distinguishing total coloring of indifference graphs. Matemática Contemporânea, 39:101$110,2010$. 
[20] V. Pedrotti and C. P. de Mello. Minimal separators in extended p4laden graphs. Discrete Applied Mathematics, 160:2769-2777, 2012.

Atílio Gomes Luiz

Quixadá - CE

Universidade Federal do Ceará

Campus Quixadá

gomes.atilio@ufc.br

José Francisco Mota Oliveira

Mogi Mirim - SP

Escola Estadual Professor Ernani

Calbucci

mota.jf@hotmail.com

Renata de Lacerda M. Vulcani

Mogi Guaçu - SP

Escola Estadual Padre Armani

DE Mogi Mirim

vulcanirenata@gmail.com

Simone Dantas

Niterói - RJ

Universidade Federal Fluminense IME - Campus Gragoatá

sdantas@id.uff.br
Christiane Neme Campos

Campinas - SP

Univ. Estadual de Campinas

Instituto de Computação

campos@ic.unicamp.br

Luis Arturo Perez Lozada

Campinas - SP

luisarturo.perezlozada@gmail.com
Sheila Morais de Almeida

Ponta Grossa - PR

Univ. Tec. Federal do Paraná.

Dep. Acadêmico de Informática

sheilaalmeida@utfpr.edu.br

Vagner Pedrotti

Campo Grande - MS

Univ. Fed. do Mato Grosso do $\mathrm{Sul}$

Faculdade de Computação

pedrotti@facom.ufms.br 